\title{
User-inspired design methodology using Affordance Structure Matrix (ASM) for construction projects
}

\author{
J. Uma Maheswari ${ }^{1, *}$, V. Paul C. Charlesraj ${ }^{2}$, and Soma Battacharya ${ }^{3}$ \\ ${ }^{1}$ Indian Institute of Technology Delhi, New Delhi 110016 India \\ ${ }^{2}$ RICS School of Built Environment, Delhi NCR 201313 India \\ ${ }^{3}$ Samsung Heavy Industries, Noida, Uttar Pradesh 201304 India
}

\begin{abstract}
Traditionally, design phase of construction projects is often performed with incomplete and inaccurate user preferences. This is due to inefficiencies in the methodologies used for capturing the user requirements that can subsequently lead to inconsistencies and result in non-optimised end-result. Iterations and subsequent reworks due to such design inefficiencies is one of the major reasons for unsuccessful project delivery as they impact project performance measures such as time and cost among others. The existing design theories and practice are primarily based on functional requirements. Function-based design deals with design of artifact alone, which may yield favourable or unfavourable consequences with the design artifact. However, incorporating other interactions such as interactions between user $\&$ designer is necessary for optimised end-result. Hence, the objective of this research work is to devise a systematic design methodology considering all the three interactions among users, designers and artefacts for improved design efficiency. In this study, it has been attempted to apply the theory of affordances in a case project that involves the design of an offshore facility. A step-by-step methodology for developing Affordance Structure Matrix (ASM), which integrates House of Quality (HOQ) and Design Structure Matrix (DSM), is proposed that can effectively capture the user requirements. HOQ is a popular quality management tool for capturing client requirements and DSM is a matrixbased tool that can capture the interdependency among the design entities. The proposed methodology utilises the strengths of both the tools, as DSM compliments HOQ in the process. In this methodology, different affordances such as AUA (Artifact-User-Affordance), AAA (Artifact-ArtifactAffordance) and DDA (Designer-Designer-Affordance) are captured systematically. Affordance is considered to be user-driven in this context that is in contrast to prevailing design methodologies. The initial feedback from industry on the proposed ASM-based design methodology is encouraging.
\end{abstract}

\footnotetext{
* Corresponding author: umapaul@,civil.iitd.ac.in
} 


\section{Introduction}

A genuine question which arises in modern days to design science is: "Is design only a predictive input-output process?" In other words, "Is it sufficient to have the archetypal design where an end result is certain at the end of execution of a defined algorithm?" The answers to these questions are no longer "Yes" and a broader arena of design science is an area of modern day research. A designer designs the product by considering all functional requirements, which describe closed system only without considering user actions. But same product can cause unexpected behaviour when it enters into the environment. Hence, before entering into the construction phase, the design has to be checked for positive and negative user actions. These user actions are nothing but "affordance". Affordance is what one system interacts with another system (user, artifact). Therefore, design earlier, which was treated as a closed system design, should now be considered as an open system design (involving environment). Complex interactions arise between users and artifacts when open system design is considered. Wegner [1] established a proof showing that "interaction is an inherently more powerful paradigm for computing than algorithms". Hence, in addition to algorithmic based design approaches, user action based design, which is also known as "affordance based design" should also be considered at design phase [2]. Many theories and methodologies have evolved in order to cater to the modern day design requirement. When it comes to construction design, the requirement is even complex and amorphous.

Therefore, affordance based design, which supports such complex and open system design has been considered for investigation for the design of construction projects. Unlike functional design, affordance based design captures two way relationships. Thereby, its suitability was tested in an ongoing project involving the design of Solid Control Room/Shale Shaker Room as part of an offshore project. The complex and interactive feature of construction design has been represented in the form of an Affordance Structure Matrix (ASM). With the help of a real life case study, an endeavour has been made to represent various interactions and a path forward for the downstream design has been proposed.

\section{Background}

\subsection{Design changes}

The biggest challenge which the construction industry is facing as of today is change management. The changes that are surfaced during the execution stage has a great bearing on quality, schedule and the cost of any project. The root cause analysis of the major changes can be greatly attributed to the poor design. One can say that the main reason for this could be, there is no systematic and scientific process for design and design management, which is being followed for construction design. Till today, construction design is more of experience oriented rather than any process orientation. It is mainly human driven and so it is error prone. The construction design can be considered as a conglomeration of many subsystems. So, in simple terms, in the definition of construction design, the two most important aspects are:

- System and subsystem requirements

- The interactions among subsystems and users

If we consider the timeline of entire construction project design phase as shown in Figure 1 , though the requirements are normally considered in the initial part of the design stage of the project, as the requirements understood completely, various teams starts interacting each other in order to generate a solution to the given problem. However, there is no methodology to capture these interactions which are an integral part of a complex system design like that of construction design. A greater need is to find out a single platform or tool which can 
simultaneously cater to both requirements and system interactions and thereby generate design guidelines, which will ultimately result in a better design and thereby minimizing the cost and schedule impact of construction projects. Affordance based design is a methodology which is built around considering functions (requirements) as well as every type of interactions present in the system. The suitability of this methodology is tested with a real life example.

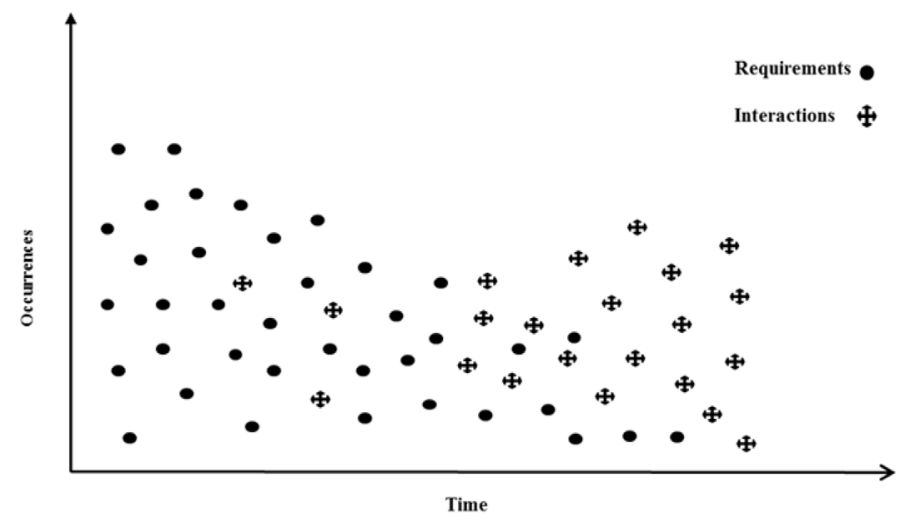

Fig. 1. Timeline of design phase of a construction project.

\subsubsection{Affordance}

The word "Affordance" does not exist in the Oxford dictionary. However the genesis of Affordance is the word "afford" and in functional sense it means "To afford". Different researchers and scientists have defined affordance in their own terms and understanding as follows:

Affordance is "Perception of the environment leads to a course of action" [3];

Affordance can be defined as "the actionable relation between the environment and an actor" (a person or animal or an object/artifact) [4];

"Affordances are properties of the environment taken relative to an observer."[5].

In the perspective of design, Affordance can be defined how a function is interacting with its surroundings. Maier [6], has put forward few adjectives which connot be affordance in design. According to him a design can't be separated from certain attribute like semantic (by literal), semi-rational, fuzzy-algorithmic, predicative, subjective, and unpredictable nature of humanity, because everything is always designed for human use, usually designed by humans themselves), and situated within an extended context of a complex world.

Artifact - An object which is not naturally present but occurs as a result of the preparative or investigative procedure is termed as artifact in general in design. In simpler term artifact what we are interested to design or what is the desire of the end user.

User - The ecological surroundings where and artefact will be used or operated consists of its users. The users could be the human who will be personally using the product, the environment where it is situated, the surrounding objects which might interact with it in the course of its operation are all considered as the user for that particular artifact.

Artifact-Artifact Affordance (AAA) - In a complex multi artifact system the different artifacts need to remain in self-organized, ordered and provide all the affordance to each other. They shall be in harmony and in an equilibrium in order to achieve a sustainable design. All requirements which one artifact has to provide to each others are defined as the artifact-artifact affordance. In construction design this definition finds an important significance as AAA is the core of construction design. AAA has a great significance in 
construction design as there are extensive numbers of interactions in construction design. Hence, it is imperative that this interaction is considered at an early stage and due importance is given at each and every stage of design. The design improvements and identifying the area of improvements can be identified from the AAA relationship. The essence of affordance is reciprocities. Hence, when we consider AAA, it actually invokes the reciprocity or the tradeoff of design, which brings about an improved design. The number of such interactions is captured in early design stage would result into a refined and improved design and execution. AAA expresses a relationship that is indirectly useful to users. Floors must support users walking on them, however, walls must support roofs, but this is ultimately to protect users within the building. AAA is significant when there is a multi-designer interface. It is of great importance that at least the AAA are identified so that designers are in a position to identify the area where this interaction can be reviewed. In case, if there are any negative affordances and the artifacts are not in harmony and equilibrium, then this can be investigated and negative affordances can be mitigated by making design improvements. For ascertaining this requirement, it is important to identify how the artifacts are related to one another and how best these relations can be established. In construction design, these play an inescapable feature and can be the leading factor for increasing design efficiency.

Artifact-User Affordance (AUA) - The essence of affordance primarily focuses on the user and endeavors to identify and maximize the artifact user affordance. AUA is the expectations or the integrated functionality the artifact provides to its users in the entire life cycle of the artifacts. A better and improved design is the one where there is an overall increase in the affordance. This could be achieved by three ways. First, ideate new and additional affordances. Second, is to improve the value of the existing positive affordances without minimizing other positive affordances. Third, is to decrease the effect of negative affordance. Figure 2 indeed shows in simple terms how four artifacts are offering the affordance to each other as required by extending the puzzle semicircle and by a cut-out on the puzzle body. Similarly, this artifact A, B, C and D can be held by the user P, Q, R and S conveniently to finally make the Jigsaw complete.

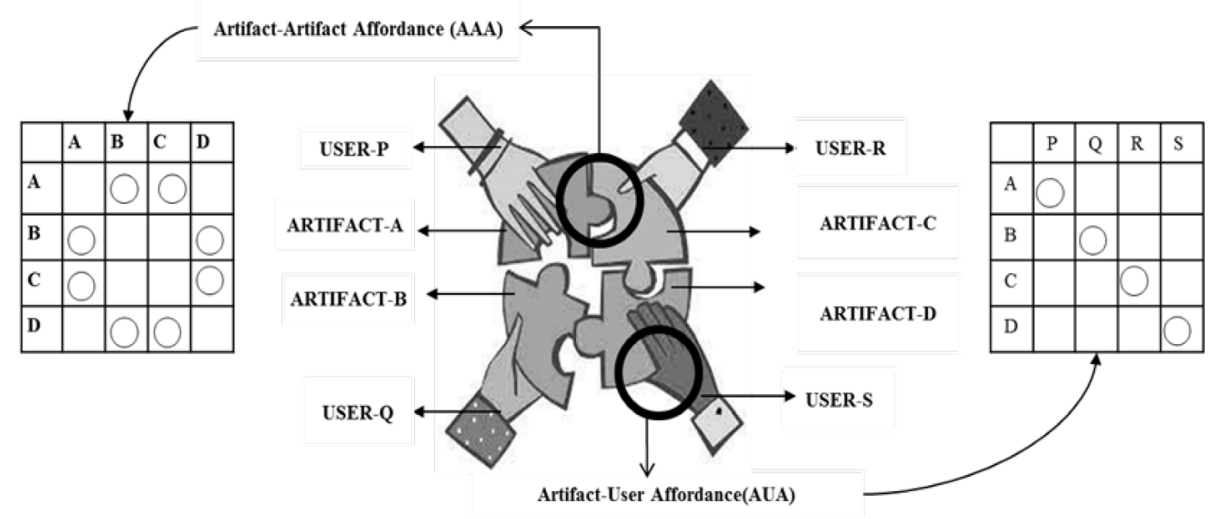

Fig. 2. AAA and AUA.

\subsubsection{Affordance structure matrix (ASM)}

All underlying theories and concepts ultimately need to evolve as an implementable form. Affordance Structure Matrix (ASM) developed by Maier et al [7] is that very form, which embodies the concepts of affordance and inspired by matrix tools of House of Quality (HOQ) and Design Structure Matrix (DSM) as shown in Figure 3. The ASM is a tool to represent 
interactions and compare requirements information with physical structure at the conceptual stage. Requirements information on design is termed as the affordance of the systems.

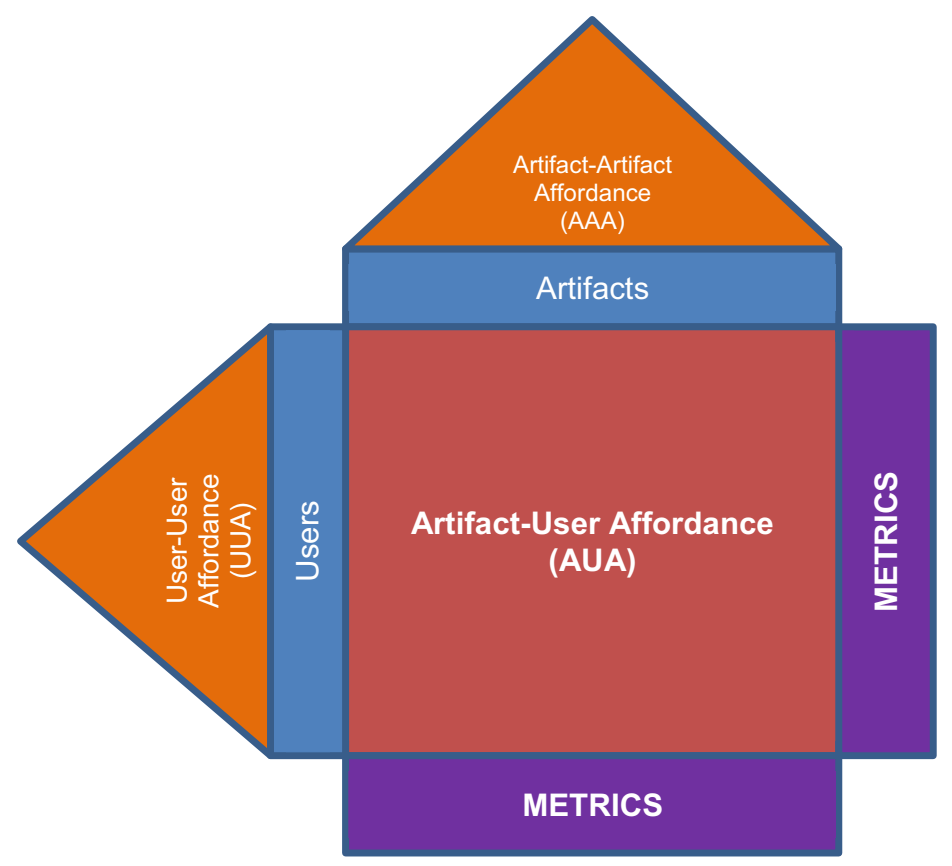

Fig. 3. Generic Representation of ASM.

The top part or the "Roof of HOQ" of the ASM is a Design Structure Matrix (DSM) that depicts the relationships among components. This is the most important part as this depicts AAA. Since these interactions play a significant role in the design of a construction project, building this metric is one of the prime steps in developing the ASM. The left side of the ASM similarly captures the relationships between affordances. However the interaction matrices and their organizations may vary depending upon the design problem and its complexity. The utility of ASM is that it is a good platform to represent various interactions and also it is possible to numerically represent those interactions.

\section{Solution methodology}

The traditional flow chart of affordance based design developed by Maier and Fadel [8] has been modified to suit the requirement of construction design and presented in Figure 4. The flow of execution this methodology is matched with the construction project design requirements. The step by step process to be followed, starting from the desire and subsequent steps have been shown in the Figure 4. The generic affordances can further be refined on specific design cases. However, these are some requirements, which are generic and is applicable in almost all types of construction design. This can be further be expanded and all micro level affordances can be identified and depicted for considering them into the design.

It is imperative that some steps may not be exactly relevant in some cases, however, the options can be evaluated in case to case basis. A systematic method to identify affordances using the interaction between functions and tasks that shall be performed by the users have been introduced in ASM for evaluating what affordances are embedded in each component 
of a product and thus grading. This matrix can illustrate correlations of affordances and also of components. This modified methodology is suitable for the construction design where multi artifact interactions are of prime importance. It is taken care that all different kinds of interactions up to the designer level has been captured into the methodology so that the design cycle is complete.

\section{Case study}

An ongoing offshore project was considered for demonstration. This project is involving the construction of a jackup rig, which is a type of mobile platform primarily used for drilling operations in the sea for an oil major of Norway that had intended to develop a jackup rig facility in the Norwegian Continental Shelf (NCS). The main study is on a "Solid Control Room/Shale Shaker Room" which is a component of jackup rig. The shale shaker room (SS room) was in its design stage and will be soon resuming its fabrication phase. SS room used as solid control system to separate solids from drilling mud. Drilling mud is used for drilling process on sea bed which is to lubricate and cool the drill bit. However, while drilling, solids generating from seabed cutting obstructs the drilling process which has to separate in shale shaker machine and reuse the drilling mud. Hence, many interactions are developed between users and machine (Artifact) inside SS room and artifact to artifact in this operation. Bad design results in inefficient operations. Thus, during design phase using affordance based design approach, all interactions have to be captured for better operations at execution phase. 

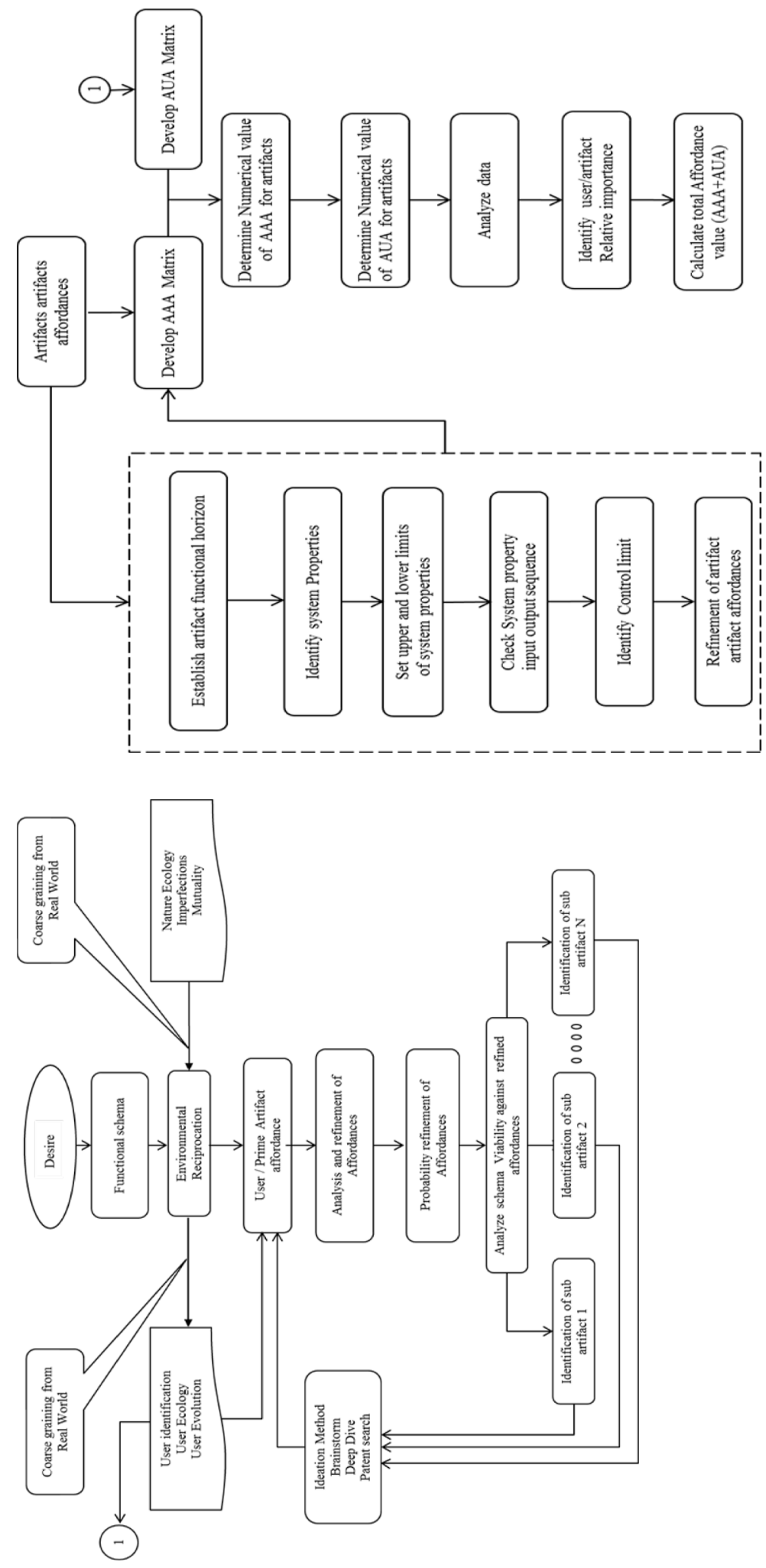

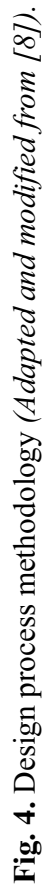


The major function of the main artifact, which is a solid control room, where solid required/generated in drilling operation will be handled. This facility comprises of many subartifacts, which are listed as a part of data collection. Data was collected by meeting the users (operators) in the course of design review and discussed on the affordances and what is crucial to them. The list of artifacts was found as part of the earlier design and form the affordances. The second set of data was collected by visiting the owner's consultant office. The project details were reviewed. Based on the discussions with engineers involved, the final users required for case study were finalized. List of affordances was prepared with the help of generic list of affordances and also discussion with the owner's consultant team and finalised.

\subsection{ASM testing and results}

The step by step process for ASM development is presented in this section, which is based on the solution methodology proposed earlier (Figure 4).

\subsubsection{Steps for development of ASM}

\section{Step 1: Desire}

Design phase begins with clients or users "Desire ". In this case study, desire is design of a SS room of a jackup rig drilling platform.

\section{Step 2: Functional schema}

A SS room to house six solid controls namely shale shaker machine (SS machine) with all its accessories with entry, exit and to enable the machine to operate for a life of 25 years. While deciding the functional schema of SS room, more information has to be coarse grain from the real world to check whether any artifacts available in the market performing the same function. If so, then how it is functioning and take reference of the same for deciding the functional schema and define the functional schema as shown in Fig. 5.

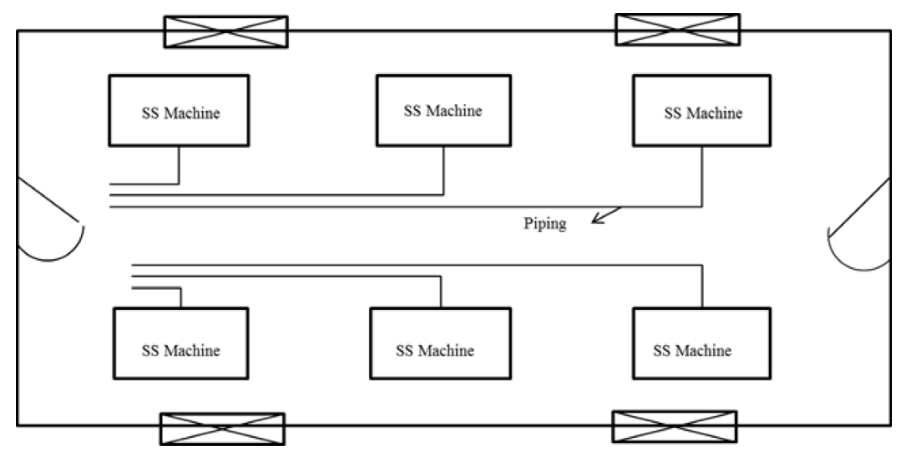

Fig. 5. Functional schema of SS room.

\section{Step 3: Environmental reciprocation}

While designing the artifact, designers have to check in what environment this artifact is finally going to build and use thereafter. Therefore, the surroundings mainly consist of the user of the room, all other physical entities around the room and the environment as it is. Here, it is a marine environment and it is possible to design a room in this environment with its users, hence there is a positive reciprocation of environment. While considering the environmental reciprocation, designer has to check the following :

- Mutuality (Can such a room coexist in this environment mutually without harming each other?); 
- Ecology (Will the ecological change allow this artifact's sustainability ?); and

- Imperfection (Consider that the functional schema decided in step 2 is not perfect and needs improvement).

Step 4: User identification

The ultimate aim of any design is how well the human can use artifact functionally. Therefore, list of users as shown in Table 1 has to be identified at design phase itself to understand their needs. Many methods are available such as by ideation, deep drive, and brainstorm methods to identify users.

Table1. List of users

\begin{tabular}{ll}
\hline S. No & Users \\
\hline 1 & Drilling Operator \\
2 & Maintenance User \\
3 & HVAC Maintenance User \\
4 & Electrical Maintenance User \\
5 & International Maritime Authority \\
6 & NORSOK \\
7 & Owner \\
8 & Fabricator \\
\hline
\end{tabular}

\section{Step 5: User Artifact Affordance}

At design phase, all users were asked for the specific requirement and what needs to be afforded by the artifacts are collated as shown in Table 2.

Step 6: Analysis and refinement of affordances

Are all the affordances viable, affordable, and justifiable? If yes, then proceed. If not, then refine the affordances and prepare the affordances accordingly. For example, is it sufficient if the SS machines were accessible from three sides, if yes then change the affordance no 1 accordingly.

Step 7: Probabilistic refinement of affordances

Are all the requirements expected in the lifetime of the artifacts? If not refine the affordances accordingly. For example, what is the probability of fire hazard? If the probability is very low eliminate requirement of passive fire protection (S. No 11 in Table 2) and keep only active fire protection arrangement.

Step 8: Analyse schema viability against refined affordances

Once all affordances are defined and refined, designer has to check again whether the functional schema is still valid for all the affordances. For instance, as discussed in step 6, slight modification in affordance no. 1 is done (accessibility made only from three sides only) and the affordance no. 11 as mentioned earlier in step 7 has been removed. 
Table 2. List of User-Artifact Affordances.

\begin{tabular}{|c|c|c|c|}
\hline S.No & User-Artifact Affordances & S.No & User-Artifact Affordances \\
\hline 1 & $\begin{array}{l}\text { All SS machines are to be accessible from } \\
\text { every side }\end{array}$ & 17 & $\begin{array}{l}\text { One side of the room will have a ballast } \\
\text { tank. Pressure for the same to be } \\
\text { afforded }\end{array}$ \\
\hline 2 & Diverter house box to be accommodated & 18 & Pipe supports can be installed \\
\hline 3 & $\begin{array}{l}\text { Access to be provided to the diverter } \\
\text { house }\end{array}$ & 19 & Weight to be within prescribed limit \\
\hline 4 & Noise level outside - less than $60 \mathrm{Db}$ & 20 & $\begin{array}{l}\text { Escape shall be minimum } 2.3 \text { meter } \\
\text { height as per norsok s002 }\end{array}$ \\
\hline 5 & Life - 25 years & 21 & $\begin{array}{l}\text { Solas (safety of life at Sea) requirement } \\
\text { met }\end{array}$ \\
\hline 6 & 2 Door size $2.5 \times 3 \mathrm{~m}$ & 22 & $\begin{array}{l}\text { Satisfy PSA (Petroleum safety } \\
\text { Authority) requirements }\end{array}$ \\
\hline 7 & Provide adequate ventilation & 23 & $\begin{array}{l}\text { NMA (national Maritime Authority) } \\
\text { regulation to be met }\end{array}$ \\
\hline 8 & Provide adequate illumination & 24 & Local law requirement to be met \\
\hline 9 & $\begin{array}{l}\text { Trolley movement within room to be } \\
\text { afforded }\end{array}$ & 25 & Economy/cost to be optimized \\
\hline 10 & $\begin{array}{l}\text { Dynamic loading due to trolley movement } \\
\text { to be afforded }\end{array}$ & 26 & $\begin{array}{l}\text { Corrosion in marine environment to be } \\
\text { afforded }\end{array}$ \\
\hline 11 & Passive fire protection to be afforded & 27 & $\begin{array}{l}\text { Corrosion under insulation to be } \\
\text { avoided }\end{array}$ \\
\hline 12 & $\begin{array}{l}\text { Window to the shaker control room to be } \\
\text { afforded }\end{array}$ & 28 & Clean design to be afforded \\
\hline 13 & $\begin{array}{l}\text { A monorail with two ton capacity to be } \\
\text { accommodated }\end{array}$ & 29 & Easy cleaning to be afforded \\
\hline 14 & Two safe escape to be provided & 30 & $\begin{array}{l}\text { Vibration generated in shale shaker } \\
\text { room to be within limits }\end{array}$ \\
\hline 15 & A stretcher movement to be afforded & 31 & Welding to be afforded \\
\hline 16 & Firewater piping to be accommodated & 32 & Erection to be afforded \\
\hline
\end{tabular}

Step 9: Define the sub-artifacts based on the affordances and functional schema

Finally, sub-artifacts that have direct interactions with users and other sub artifacts have to be generated based on affordances generated previously and functional schema (shown in the Table 3).

Step 10: Establish artifacts functional horizon

From the functional schema and the affordances, matrix of artifacts can be established. For each artifacts its functions and extent of functionality is defined. For example, in this case study, the Shale Shaker machine (SS machine) has to perform the solid control activity for a certain quantity and quality of solid per day or per hour. Apart from its main function, it also needs to be accommodated and all its other affordances listed are to be defined.

Step 11: Identify system property

For each of the artifacts, the required affordances are converted to system property. For example the affordance is that all sub-artifacts are to be accommodated. Then dimension is the system property. 
Table 3. List of Sub artifacts.

\begin{tabular}{ll}
\hline S. No & Sub artifacts \\
\hline 1 & Six Shale Shaker Machines \\
2 & Two doors \\
3 & Control Room Window \\
4 & Diverter Box \\
5 & Access platform to Diverter Box \\
6 & Trolley Movement Space \\
7 & Firewater Piping \\
8 & Mud Piping \\
9 & Fire Extinguishers \\
10 & HVAC Ducts \\
11 & Electrical Trays \\
12 & Instrument Cable Trays \\
13 & 2 Ton Capacity Monorail \\
14 & Pipe/Tray/Duct Penetrations \\
15 & Noise Insulation Panel \\
16 & Lights \\
17 & Bulkheads/Decks \\
\hline
\end{tabular}

Step 12: Define system property upper and lower limits

For each system property, define what could be the upper and lower limit of the property. For example, it is established what could be the minimum and maximum dimension for the SS machine that can be accommodated in SS room.

Step 13: Check system property input/output sequence

The system property of different artifacts depends on the system output of other artifacts. For example, the size of the SS room would depend on the size of the SS machine. The output (dimension) of SS machine is the input to the size of the SS room.

Step 14: Refinement of artifact-artifact affordance

Once the artifact-artifacts affordances are defined, it may be reviewed for further refinement.

Step 15: Develop roof of ASM (AAA interaction matrix)

First of all, the sub artifacts have been considered and they have been placed in the Top Main row area as shown in Fig. 6. The roof of the ASM is considered for developing AAA interaction matrix, which is DSM of artifacts. Here it is considered how one artifact is related to other artifact.

Step 16: Develop main body of ASM (AUA interaction matrix)

All users have been listed in the left main column of ASM and related to artifacts to develop AUA interaction matrix as shown in Fig. 6. It is decided to find out how each and every user is related to different sub artifacts.

Step 17: Numerically simulate the AAA index

A numerical simulation is done by assigning relationship index (S- Strongly Related, M - Moderately Related, L- Weakly Related). Numerical value of 9, 3, and 1 has been assigned to this relationship. Total index per artifact has been calculated to establish an AAA index for that artifact. 
Step 18: Numerically simulate the AUA index

Similar relationship index has been found from data and numerical values assigned as explained in step 17. Total index per user-artifact relationship has been calculated to establish an AUA index for that artifact.

Step 19: Analyse data and identify user/artifact relative importance

From this DSM, the AAA index such as weak relation for each artifact can be found out and further improvement in the design can be made. Similar way, the weak relation between users and artifact in AUA interaction matrix can be worked out for design improvement. This step considerably increases the positive affordance as all AAA and AUA have been captured and hence, the total AAA and AUA value can increase.

Step 20: Total Affordance Index:

Total Affordance Index (Sum of AAA and AUA) calculated to find out which artifact or a group of artifacts will be the key governor and will lead the design.

\subsection{Discussion}

\subsubsection{Polarity}

Affordances have already been classified based on the type of reciprocities as AAA and AUA. However, affordances have also been classified based on their polarity. If the affordance is beneficial to the user of the artifact, it is considered to a positive affordance. In case the affordance is harmful to the user, it is considered as the negative affordance. This concept has a prime importance in the design. As a designer, we are expected to increase the positive affordance and reduce the negative affordance. This has been explained by an example in the Figures $7 \& 8$.

In Figure 7, the open window had two positive affordances, day light and ventilation. At the same time this also had a negative affordance of entry of burglar or animal. The total affordance of the design was only 1 . In the Figure 8, iron grills are provided on the window and the negative affordance has been converted to a positive affordance and the total affordance of the window design has been increased to 3. In this way, design can be improved by eliminating the negative design or providing solutions to negative design.

\subsubsection{Designer- designer affordance}

In construction design, it is imperative that when AAA exists, the relevant designer also have an affordance to other designers so that an improved design can be achieved. So from AAA matrix, affordance list and from the list of designers, the Designer-Designer Affordance (DDA) matrix can be generated. The relative importance of the designer can be established from the numerically simulated index value. From the DDA matrix, input and output requirement of parameters, relevant design documents can be scheduled. DSM techniques may apply for efficient scheduling of the documents. 


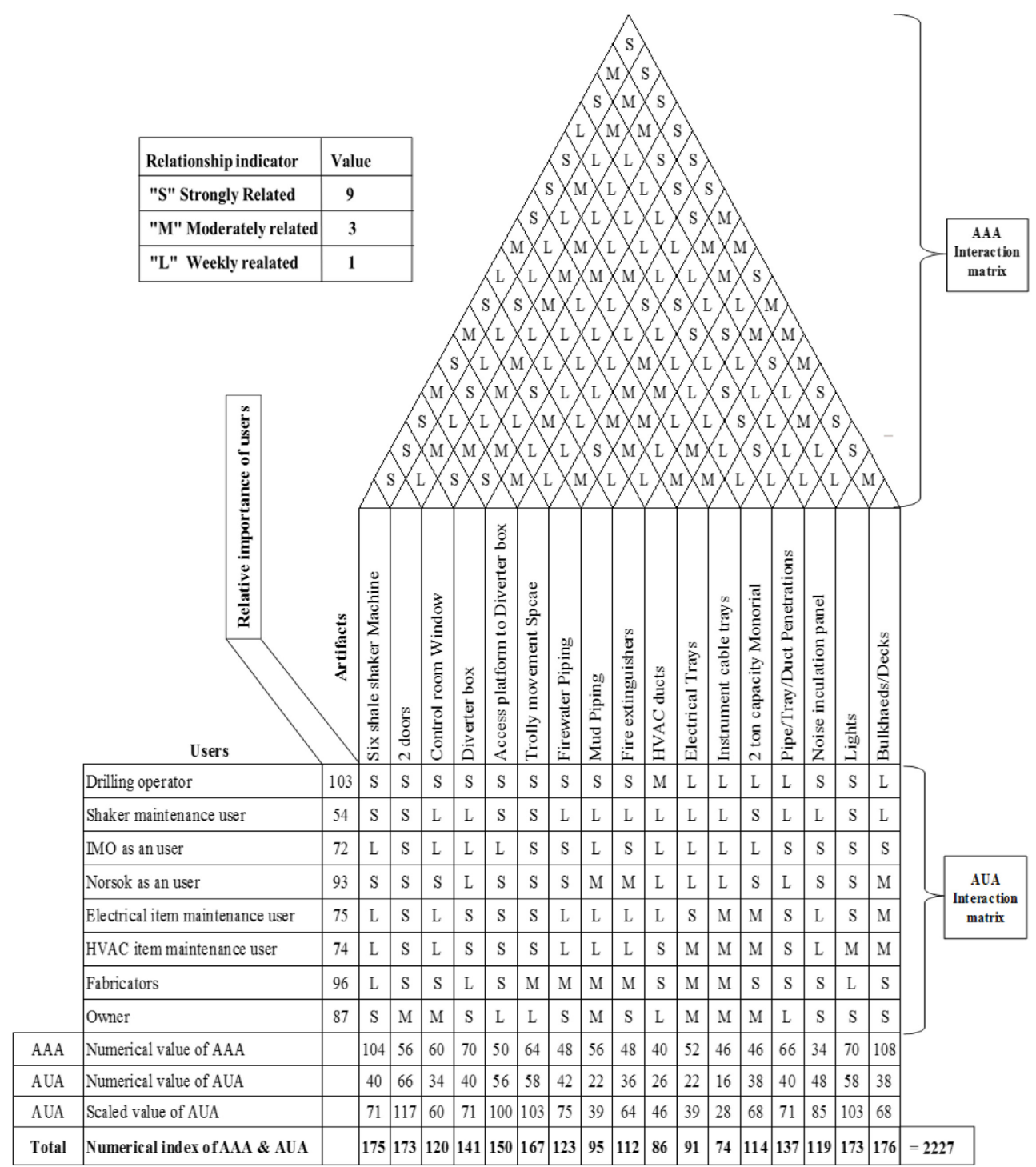

Fig. 6. ASM for a Shale Shaker Room.

Note:

A lower index of AAA matrix can have a higher value of AUA; A lower value of the AUA index can have a higher index of $A A A$. 

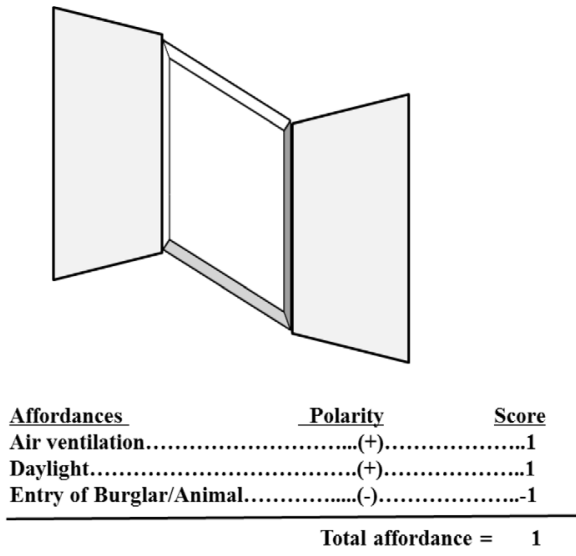

Fig 7. Design with negative affordance.
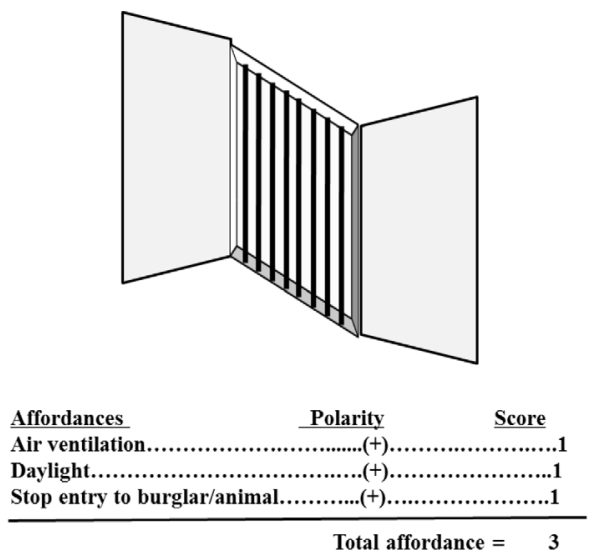

Fig. 8. Change of negative to positive affordance.

\section{Conclusion}

The concept of affordance definitely paves a way for a systematic and successful methodology, which can be implemented in construction design. The early capture of all affordances and the concept of AAA, AUA and DDA in DAU (Designer-Artifact-User) System adds a process approach to the conventional design hierarchy. The simple understanding that increases in positive affordance will yield a better design can be acceptable. However, whether this methodology is the best approach compared to other concepts that are on-course is questionable. Expert's comments align with this concept. Time required for this approach would be a hindrance to the acceptance of this process. But implementing this process can substantially lead to efficient design for construction projects. The salient conclusions are:

- The concept of affordance initiates the process of identifying expectations /requirements from a design

- The ASM framework enables capturing all interaction and relations by matrix approach and providing a common language among designers

- Systematic trading off of affordance (increasing positive affordance and decreasing negative affordance) yields an alternate design

- Micro level design improvement, where multi artifacts and multidisciplinary design is involved can be achieved by AAA and DDA

- This methodology also facilitates information cataloguing, leading to archived knowledge for use in future projects.

\section{References}

1. P. Wegner, Communications of ACM, 40, 80 (1997)

2. J. R. A. Maier, and G. M. Fadel, Research in Engineering Design, 20, 13 (2009)

3. J. J. Gibson, in: Shaw, R. and Bransford, J. (Eds.), Perceiving, acting, and knowing. toward an ecological psychology, 67 (Lawrence Erlbaum Associates, Hillsdale, NJ, 1977)

4. J. J. Gibson, The ecological approach to visual perception, (Pyschology Press, New York, 1986)

5. A. J. Wells, Ecological Psychology, 14, 140 (2002) 
6. J. R. A. Maier, Affordance based design: theoretical foundations and practical applications (VDM Verlag Dr. Muller GmbH \& Co, Saarbrucken, Deutschland, 2011)

7. J. R. A. Maier, T. Ezhilan, and G. M. Fadel, Proceedings of the ASME 2007 International Design Engineering Technical Conferences and Computers and Information in Engineering Conference, 277 (2007)

8. J. R. A. Maier, and G. M. Fadel, Proceedings of ICED 2007, the 16th International Conference on Engineering Design (2007) 